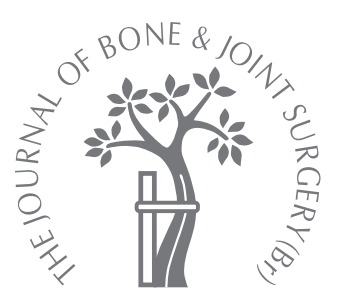

M. L. Costa,
K. MacMillan,
D. Halliday,
R. Chester,
L. Shepstone,

A. H. N. Robinson,

S. T. Donell

From The University

of East Anglia, Cambridge and The

Norfolk and

Norwich University

Hospital, Norwich, and Addenbrooke's

Hospital, Cambridge, England

M. L. Costa, PhD, FRCS Orth, Honorary Lecturer in Orthopaedics

School of Medicine

K. MacMillan, MCSP, Senior

Physiotherapist

D. Halliday, MCSP, Senior

Physiotherapist

Department of Physiotherapy

R. Chester, MSc, Lecturer in

Physiotherapy

School of Allied Health

Professionals

ㄴ. Shepstone, PhD, Reader

in Medical Statistics

S. T. Donell, MD, FRCS

Orth, Honorary Reader in

Orthopaedics

School of Medicine

University of East Anglia,

Norwich NR4 7TJ, Norfolk,

UK.

A. H. N. Robinson, FRCS

Orth, Consultant Orthopaedic

Surgeon

Addenbrooke's Hospital, Hills

Road, Cambridge CB2 300,

Cambridgeshire, UK.

Correspondence should be sent to Mr M. L. Costa; e-mail: mattcosta@hotmail.com

(C)2006 British Editorial

Society of Bone and

Joint Surgery

doi:10.1302/0301-620X.88B1.

$16549 \$ 2.00$

$J$ Bone Joint Surg [Br]

2006;88-B:69-77.

Received 30 March 2005;

Accepted after revision

13 September 2005

\title{
Randomised controlled trials of immediate weight-bearing mobilisation for rupture of the tendo Achillis
}

\begin{abstract}
We performed two independent, randomised, controlled trials in order to assess the potential benefits of immediate weight-bearing mobilisation after rupture of the tendo Achillis. The first trial, on operatively-treated patients showed an improved functional outcome for patients mobilised fully weight-bearing after surgical repair. Two cases of rerupture in the treatment group suggested that careful patient selection is required as patients need to follow a structured rehabilitation regimen. The second trial, on conservatively-treated patients, provided no evidence of a functional benefit from immediate weight-bearing mobilisation. However, the practical advantages of immediate weight-bearing did not predispose the patients to a higher complication rate. In particular, there was no evidence of tendon lengthening or a higher re-rupture rate. We would advocate immediate weight-bearing mobilisation for the rehabilitation of all patients with rupture of the tendo Achillis.
\end{abstract}

Rupture of the tendo Achillis is a disabling condition with an incidence of 18 per 100000 per year; ${ }^{1}$ this incidence is increasing. ${ }^{2}$ The condition typically affects young active adults and is associated with prolonged periods off work and sporting activity. ${ }^{3}$ Consequently, delayed rehabilitation has significant financial and health implications.

There are two key problems which delay rehabilitation following rupture of the tendo Achillis: 1) healing of the tendon, a slow process during which there is a risk of rerupture under load; and 2) muscle atrophy associated with immobilisation. Disuse atrophy occurs very quickly and is difficult to reverse. Traditional rehabilitation from an injury to the tendo Achillis has, therefore, involved prolonged protection of the tendon, usually in a plaster cast, followed by extensive physiotherapy to regain the lost function of the calf muscles. Experimental models suggest that early loading of the ruptured tendo Achillis prevents detrimental alterations in muscle characteristics and favourably influences maturation of collagen fibres within the tendon., As a result, controlled early loading and movement may be beneficial.

Our hypothesis was that immediate weightbearing would produce faster rehabilitation for patients after a rupture of the tendo Achillis. We therefore devised two trials in order to compare immobilisation involving immediate weight-bearing with traditional non-weightbearing. Trial 1 assessed patients who had been treated operatively and trial 2 assessed those treated non-operatively. The aim was to improve rehabilitation and hasten return to normal activity for patients with a rupture of the tendo Achillis.

\section{Patients and Methods}

The patients were recruited from three orthopaedic centres, The Norfolk and Norwich University Hospital, Addenbrooke's Hospital and Colchester General Hospital, between January 2001 and November 2002. The Local Research Ethics Committee at each hospital gave their approval for the study.

All patients had ruptured their tendo Achillis within the previous seven days. The diagnosis was made clinically, with ultrasonographic confirmation if there was any doubt. Each patient chose either operative or non-operative treatment in consultation with the admitting team, before discussing the trial. There were 52 patients who decided on operative treatment and who were then approached to enter trial 1. Four declined. The remaining 48 gave informed consent. There were 48 patients who decided on non-operative treatment and who then gave informed consent to enter trial 2; no patient in this group declined to take part. This sample size was calculated from the results of a pilot study. ${ }^{6}$ 


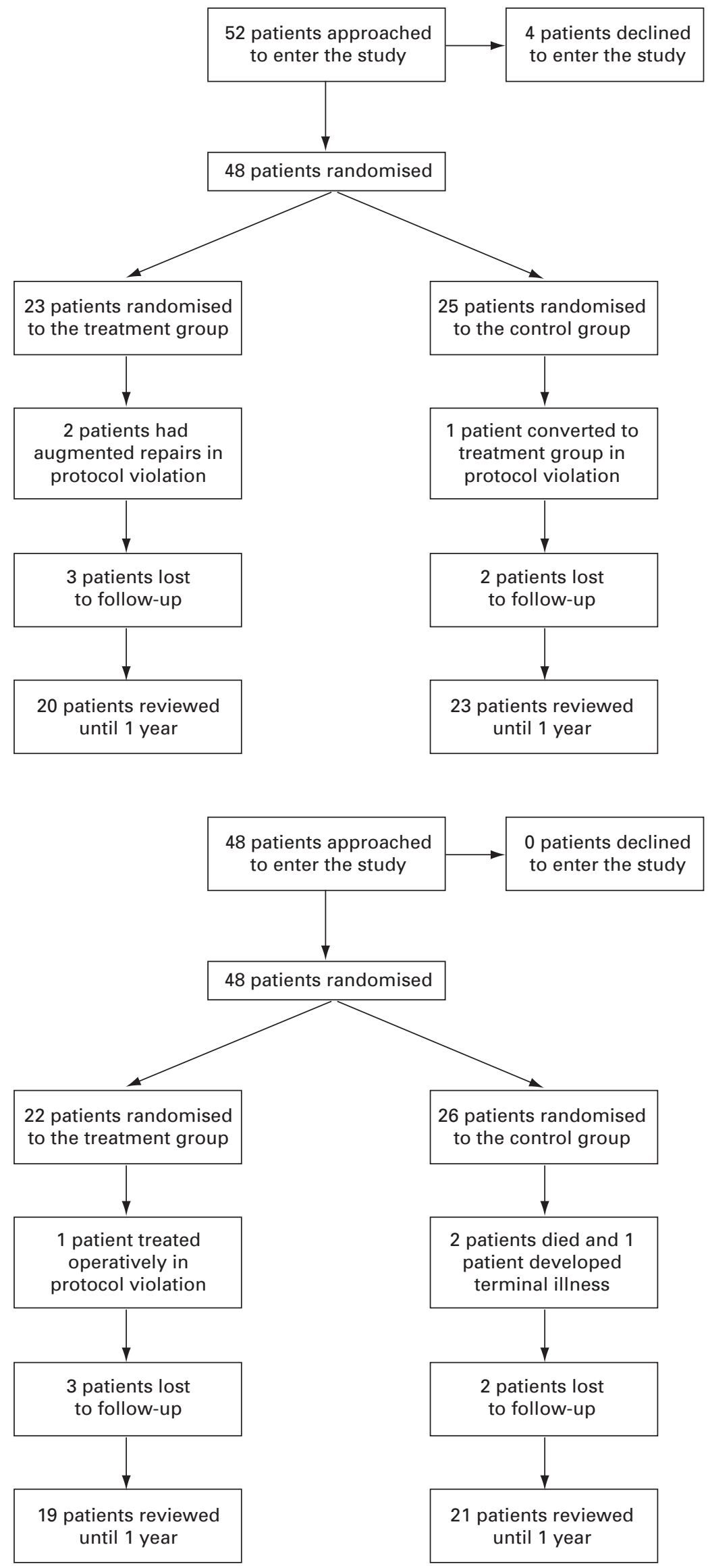

Flow summary for patients treated a) operatively and b) non-operatively.
Fig. 1b 


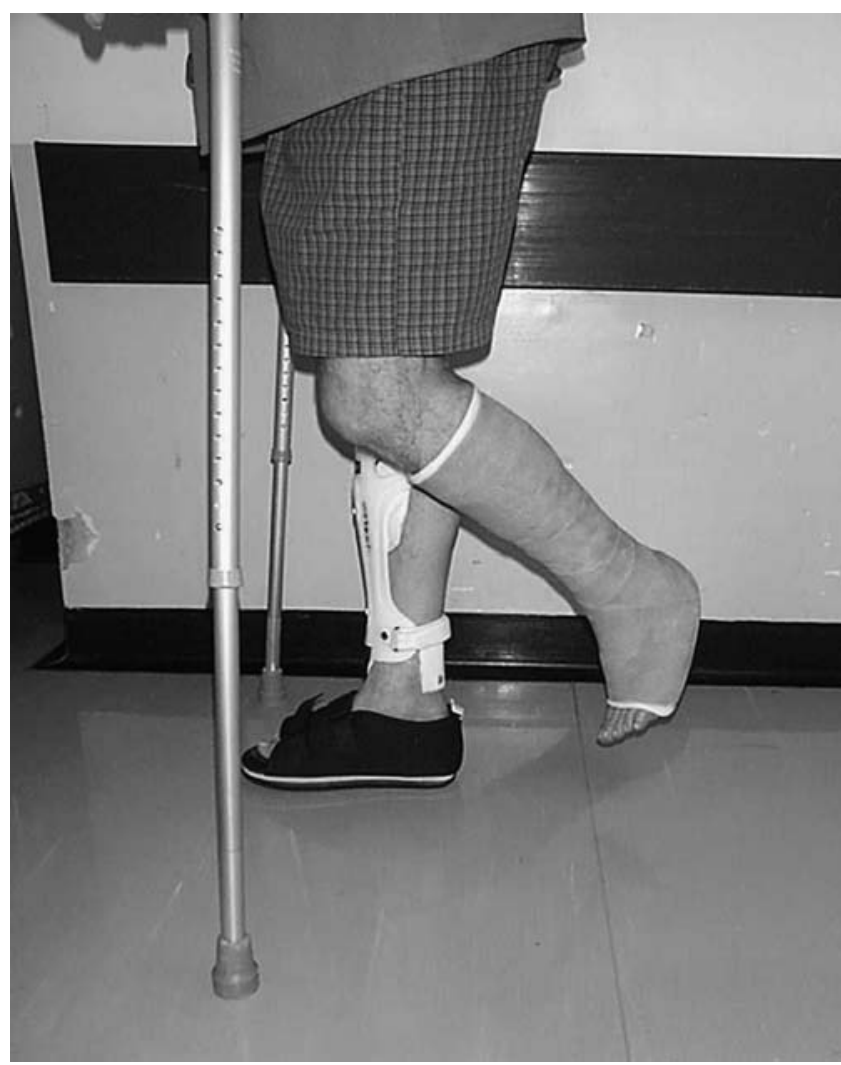

Fig. 2

Full weight-bearing mobilisation in an orthosis compared with nonweight-bearing in a plaster cast (patient with bilateral ruptures).

Operative repair. Two of the patients treated operatively had complex ruptures at operation, one involving a massive calcified tendinosis and the other a partial avulsion from the calcaneum. Both had augmented repairs, with a tendon lengthening procedure and bone anchors, respectively. Their rehabilitation was delayed, but both were followed up on an intention-to-treat basis within their allocated treatment group. The remaining 46 patients had a simple, open end-to-end operative repair performed under general anaesthesia. The incision and exact technique of repair were left to the discretion of the operating surgeon. The limb was then placed in a temporary gravity equinus plaster overnight.

Randomisation was performed using computer-generated random numbers which were produced before the start of the trial. These were sealed in sequentially-numbered envelopes, which were opened after each patient consented to take part. Patients were randomised to either the treatment group for immediate mobilisation in a carbonfibre orthosis with three $1.5 \mathrm{~cm}$ heel raises (Tyco, Gosport, UK), or the control group, who had traditional plaster cast immobilisation. Patients who had been treated operatively began their rehabilitation on the first post-operative day. Operative follow-up. At the first follow-up all wounds were assessed and sutures removed. Patients were then clinically reviewed every two weeks for eight weeks. The equinus position of the plaster or the number of heel raises in the orthosis was reduced at each visit until a plantigrade position of the ankle was achieved. The orthosis or plaster cast was removed at eight weeks.

Loss to follow-up (operative). Five patients were lost to follow-up in trial 1 , leaving 43 with complete data sets at one year (Fig. 1a). However, only one patient was lost before returning to normal activities. The remaining four all moved from the area without leaving forwarding addresses, but had returned to normal activities before being lost from the study.

Non-operative management. The non-operatively managed patients who presented during normal working hours were randomised immediately. Those who presented out of hours were placed in a temporary below-knee equinus plaster backslab and asked to attend the outpatient department the following Monday for randomisation.

Patients in the control groups were placed into a belowknee gravity equinus cast and mobilised non-weight-bearing. The treatment groups were fitted with the same off-theshelf, carbon-fibre orthosis with three $1.5 \mathrm{~cm}$ heel raises. They were then mobilised with full weight-bearing and encouraged to move the ankle within the orthosis (Fig. 2). Non-operative follow-up. The equinus position of the hindfoot was not changed for the first six weeks. The position of the plaster cast or the number of heel raises in the orthosis was then reduced at two-weekly intervals before being removed at 12 weeks. A written exercise programme was given to each patient. The rehabilitation regimen was a series of exercises performed sequentially over the six months after injury; no formal physiotherapy was offered. Loss to follow-up (non-operative). Five patients were lost to follow-up in trial 2 although only two were lost before they had returned to normal activities. In addition, two patients died during the study and one had a serious, unrelated illness and was too unwell to contribute after four months of review. This left 40 patients with complete data sets at one year (Fig. 1b).

Patient evaluation. Each patient was subsequently reviewed at three months, six months and one year. The primary outcome measure was the time taken to return to normal activities, as reported by the patient. Data relating to the return to normal sporting activity, walking, stair climbing and work activity were also recorded. In addition, the EuroQol health status questionnaire, whch has two domains, the E5D dimensions of health and the EQOL health score, was completed at baseline, ten weeks, six months and one year. ${ }^{7}$ At the six-month review, an independent physiotherapist who was blinded to the patients' treatment, performed a clinical assessment and measured calf muscle function using the Kincom 125E Plus Dynamometer with version 5.20 software (Chattanooga Group Inc, Hixson, Tennessee). ${ }^{8}$

Analysis. The primary outcome, the time to return to normal activities, was analysed using a log-rank test. The num- 
Table I. Demographics for the operatively-treated patients

\begin{tabular}{lll}
\hline & $\begin{array}{l}\text { Treatment group } \\
\text { (n= 23) }\end{array}$ & $\begin{array}{l}\text { Control group } \\
\text { (n= 25) }\end{array}$ \\
\hline Age in yrs (range) & $42(28$ to 61$)$ & 42 (29 to 69) \\
Men:women & $18: 4^{*}$ & $22: 3$ \\
Manual:non-manual job & $13: 9^{*}$ & $10: 15$ \\
Right:left & $10: 13$ & $10: 15$
\end{tabular}

* where the number of patients does not equal the total number of patients randomised to each group, no data were recorded

ber of patients returning to sporting activity was analysed using Fisher's exact test. The EuroQol data sets were analysed cross-sectionally using a Wilcoxon-Mann-Whitney test. The clinical variables at six months were analysed using a two-sample $t$-test. Confidence intervals accompanied all testing of hypotheses. As this was a pragmatic trial, an intention-to-treat approach was used in all analyses. Values for $\mathrm{p}<0.05$ were regarded as significant.

\section{Results}

Operatively-treated patients. We randomised 23 patients to immediate weight-bearing mobilisation and 25 to plaster cast immobilisation. One patient was randomised to the plaster group but was converted to the orthosis at three weeks in violation of the study's protocol.

The mean age for each group was 42 years (Table I). There were similar ratios of right- to left-sided tendon ruptures in the two groups, and more men in both groups. All patients were classified as either recreational or club-level sporting individuals, except for one higher level individual in each group. ${ }^{9}$ There were more manual workers in the treatment than in the control group.

There was a significant difference $(\mathrm{p}=0.027)$ between the groups in the time taken to return to normal walking, with a median of 12.5 weeks in the treatment group and 18 weeks for the control group. There was also a significant reduction $(\mathrm{p}=0.023)$ in the time taken to return to normal stair climbing, with a median of 13 weeks in the treatment group and 22 weeks for the controls. There was no evidence of a treatment effect in terms of time to return to sport $(\mathrm{p}=0.341)$ or work $(\mathrm{p}=0.593)$ (Table II). Four patients in the treatment group and eight in the control group did not return to their previous sporting activities and were censored at 52 weeks. The patient who was lost to follow-up before returning to normal activities was in the treatment group.

Table II. Analysis of the return to sport, walking, stair climbing and work for the patients who were treated operatively

\begin{tabular}{|c|c|c|c|c|}
\hline & Treatment group $(\mathrm{n}=\mathbf{2 3}$ ) & Control group $(n=25)$ & p value & $95 \% \mathrm{Cl}^{*}$ \\
\hline Number returning to sport $(\%)$ & $19(83)$ & $17(68)$ & 0.324 & \\
\hline \multicolumn{5}{|c|}{ Time in weeks taken to return to activities: } \\
\hline \multicolumn{5}{|l|}{ Sport } \\
\hline Number (censored) & $23 \quad(7)$ & $25(9)$ & & \\
\hline Median $(95 \% \mathrm{Cl})$ & $39 \quad(18$ to 60$)$ & $26(40$ to 90$)$ & 0.341 & -11.2 to 43.2 \\
\hline \multicolumn{5}{|l|}{ Walking } \\
\hline Number (censored) & $22(0)$ & $25(0)$ & & \\
\hline Median (95\% Cl) & 12.5 (10 to 18$)$ & $18(18$ to 22$)$ & 0.027 & 0 to 11 \\
\hline \multicolumn{5}{|l|}{ Stair climbing } \\
\hline Number (censored) & $22(0)$ & $24(0)$ & & \\
\hline Median $(95 \% \mathrm{Cl})$ & 13 (10 to 18$)$ & $22(18$ to 22$)$ & 0.023 & 5.3 to 12.7 \\
\hline \multicolumn{5}{|l|}{ Work } \\
\hline Number (censored) & $20 \quad(0)$ & $25(0)$ & & \\
\hline Median (95\% Cl) & $8 \quad(2$ to 13$)$ & $4(1$ to 13$)$ & 0.593 & -1.5 to 9.5 \\
\hline
\end{tabular}

* $\mathrm{Cl}$, confidence interval

Table III. Analysis of health scores for the patients who were treated operatively. Where the number of patients did not equal the total randomised to each group, these data were not recorded

\begin{tabular}{|c|c|c|c|c|c|}
\hline \multirow[b]{2}{*}{ Measure } & \multicolumn{2}{|c|}{ Experimental group $(n=23$ ) } & \multicolumn{2}{|c|}{ Control group ( $n=25$ ) } & \multirow[b]{2}{*}{ p value ${ }^{\dagger}$} \\
\hline & Number & Median (IQR)* & Number & Median (IQR) & \\
\hline \multicolumn{6}{|l|}{$\mathrm{EQoL}^{\ddagger}$} \\
\hline 10 weeks & 16 & (70 to 85 & 75 & (65 to 80$)$ & 0.854 \\
\hline 6 months & 21 & (80 to 90$)$ & 81 & $81 \quad$ (75 to 95$)$ & 0.956 \\
\hline 12 months & 18 & (75 to 95$)$ & 90 & $90 \quad$ (85 to 95$)$ & 0.138 \\
\hline \multicolumn{6}{|l|}{$\mathrm{E} 5 \mathrm{D}^{\S}$} \\
\hline 10 weeks & 17 & 0.69 (0.6 to 0.9$)$ & 0.69 & 0.69 (0.6 to 0.7$)$ & 0.450 \\
\hline 6 months & 21 & $0.80(0.7$ to 1.0$)$ & 0.80 & $0.80(0.8$ to 1.0$)$ & 0.956 \\
\hline 12 months & 18 & $1.00(0.9$ to 1.0$)$ & 1.00 & $1.00(0.8$ to 1.0$)$ & 0.146 \\
\hline
\end{tabular}

* IQR, interquartile range

$\dagger$ Wilcoxon-Mann-Whitney test

‡ EQoL health score, a domain of the Euroquol

$\S \mathrm{E} 5 \mathrm{D}$, dimension of health score of the Euroquol 
Table IV. Clinical and dynamometry assessments (mean (SD)) for the patients who were treated operatively six months after their rupture of the tendo Achillis

\begin{tabular}{|c|c|c|c|c|}
\hline Variable & Treatment group ( $n=19$ ) & Control group $(n=23)$ & p value ${ }^{*}$ & $95 \% \mathrm{Cl}^{\dagger}$ \\
\hline \multicolumn{5}{|l|}{ Clinical data (mean (SD)) } \\
\hline Defect in calf diameter in $\mathrm{mm}$ & $1.47(1.77)$ & $0.73(1.21)$ & 0.114 & -0.19 to 1.68 \\
\hline Loss of dorsiflexion in degrees & $1.84(4.38)$ & $(7.13)$ & 0.54 & -4.95 to 2.63 \\
\hline Loss of plantar flexion in degrees & $0.63(13.99)$ & $2.43(9.89)$ & 0.628 & -4.95 to 2.63 \\
\hline Dynamometry data (mean (SD)) & $\mathrm{n}=20$ & $\mathrm{n}=23$ & & \\
\hline Deficit in peak concentric torque $(\%)$ & $-3.51(55)$ & $18.84(39)$ & 0.131 & -51.64 to 6.93 \\
\hline Deficit in peak eccentric torque (\%) & $8.54(43)$ & $18.02(36)$ & 0.437 & -33.87 to 14.9 \\
\hline Deficit in mean concentric torque (\%) & $1.31(57)$ & $22.44(45)$ & 0.184 & -52.73 to 10.45 \\
\hline Deficit in mean eccentric torque (\%) & $7.78(52)$ & $26.89(29)$ & 0.142 & -44.87 to 6.65 \\
\hline Deficit in total concentric work (\%) & $2.63(57)$ & $23.54(43)$ & 0.176 & -51.56 to 9.75 \\
\hline Deficit in total eccentric work (\%) & $8.05(52)$ & $26.74(29)$ & 0.147 & -44.21 to 6.82 \\
\hline
\end{tabular}

* $t$-test

$\dagger \mathrm{Cl}$, confidence interval

The EuroQol health status questionnaire showed that the patients progressively improved during the study (Table III). As expected, the maximum functional deficit occurred two weeks after either the orthosis or cast was removed. The majority of the patients in both groups had returned to their pre-injury state by six months after the injury, an improvement which was maintained at one year.

There was no difference between the groups in the clinical variables at six months (Table IV). In particular, there was no difference in the range of ankle movement. Similarly, the dynamometry assessment did not show any difference in calf muscle function between the two groups.

In the treatment group, two patients re-ruptured their tendo Achillis. The first had returned to work as a boatman in his orthosis, slipped on ice and forcibly dorsiflexed his ankle. The second was running on a treadmill within two weeks of removal of his orthosis, in breach of the written rehabilitation protocol, fell off the treadmill and re-ruptured his tendon. Both patients had revision repairs and made unremarkable recoveries. A third patient, who had been randomised to the treatment group, but was treated in a plaster cast after an augmented repair, required a second operation to provide skin cover over a dehisced wound. There were also two major complications in the control group. The first patient developed persistent paraesthesiae in the foot which was treated conservatively throughout the study. A second patient stumbled while using his crutches and ruptured his contralateral tendo Achillis (Fig. 1). This was treated by operative repair and he mobilised fully weight-bearing in the orthosis. He made a good recovery.

There were six minor wound complications (including one superficial infection) in the treatment group, and five in the control group. All resolved with conservative treatment. One patient in the control group underwent a laparotomy for unrelated reasons four months after his tendon injury; this delayed his rehabilitation by a few weeks.

Non-operatively-treated patients. We randomised 22 patients to immediate weight-bearing mobilisation and 26 to plaster-cast immobilisation. One patient in the treatment group withdrew from the study after three weeks. He was subse-
Table V. Demographics for the non-operatively-treated patients

\begin{tabular}{lll}
\hline & $\begin{array}{l}\text { Treatment } \\
\text { group (n = 22) }\end{array}$ & $\begin{array}{l}\text { Control group } \\
\text { (n = 26) }\end{array}$ \\
\hline Age in years (range) & $53(21$ to 79) & 53 (31 to 79) \\
Men:women & $15: 7$ & $17: 9$ \\
Manual job:non-manual job or retired & $9: 13$ & $9: 16$ \\
Right:left side & $10: 11^{\dagger}$ & $10: 15^{\dagger}$ \\
Tendon:MTJ* rupture & $15: 6^{\dagger}$ & $20: 5^{\dagger}$
\end{tabular}

* MTJ, musculotendinous junction

$\dagger$ where the number of patients did not equal the total randomised to each group, these data were not recorded

quently treated in a plaster cast and reviewed as per the protocol, but did not attend for follow-up after four months. A second patient in the treatment group had a further minor injury after four weeks in the study. A precautionary ultrasound investigation at this stage revealed gross tendinosis and no sign of tendon healing. He had previously sustained two ruptures of the contralateral tendon. On this basis, it was decided that he should undergo a flexor hallucis longus transfer. Following this, he had a delayed rehabilitation but was reviewed in the treatment group on an intention-to-treat basis.

The mean age for each group was 53 years (Table V). There were similar ratios of right- to left-sided tendon ruptures in the two groups and more men in both groups. All of the patients were classified as either recreational or clublevel sporting individuals, except for one higher level individual in the treatment group. The number of manual workers in each group was the same. Six of the treatment group and five of the control group had ruptures at the musculotendinous junction. This was defined as being within the tendon but more than $6 \mathrm{~cm}$ proximal to the calcaneum.

The primary outcome measure showed no difference between the groups in the time taken to return to either walking $(\mathrm{p}=0.765)$ or climbing stairs $(\mathrm{p}=0.484)$ normally. In addition, there was no evidence of a treatment effect in terms of the time to return to either sport $(p=0.631)$ or 
Table VI. Analysis of the return to sport, walking, stair climbing and work for the patients who were treated non-operatively. Where the number of patients did not equal the total randomised to each group, these data were not recorded

\begin{tabular}{|c|c|c|c|c|}
\hline & Treatment group $(n=22)$ & Control group $(\mathrm{n}=\mathbf{2 6}$ ) & p value & $95 \% \mathrm{Cl}^{*}$ \\
\hline \multicolumn{5}{|c|}{ Time in weeks taken to return to activities: } \\
\hline Number (censored) & $18 \quad(10)$ & $21(13)$ & & \\
\hline Median (95\% Cl) & $-\quad(22)$ & $-(26)$ & 0.631 & Incalculable \\
\hline \multicolumn{5}{|l|}{ Walking } \\
\hline Median (95\% Cl) & 18 (12 to 22$)$ & 18 (18 to 22$)$ & 0.765 & -5 to 5 \\
\hline \multicolumn{5}{|l|}{ Stair climbing } \\
\hline Number (censored) & $16(0)$ & $22(1)$ & & \\
\hline Median $(95 \% \mathrm{Cl})$ & 17 (11 to 18$)$ & 18 (14 to 22$)$ & 0.484 & -2.6 to 4.6 \\
\hline \multicolumn{5}{|l|}{ Work } \\
\hline Number (censored) & $13(0)$ & $17(0)$ & & \\
\hline
\end{tabular}

Table VII. Analysis of health scores for the patients who were treated non-operatively. Where the number of patients did not equal the total randomised to each group, these data were not recorded

\begin{tabular}{|c|c|c|c|c|c|c|c|}
\hline & \multicolumn{3}{|c|}{ Treatment group $(\mathrm{n}=22)$} & \multicolumn{3}{|c|}{ Control group $(n=26)$} & \multirow[b]{2}{*}{ p value ${ }^{\dagger}$} \\
\hline & Number & Mec & $\operatorname{lian}\left(\right.$ IQR) ${ }^{*}$ & Number & Mec & lian (IOR) & \\
\hline \multicolumn{8}{|l|}{ EQoL } \\
\hline 14 weeks & 13 & 80 & (70 to 85 ) & 17 & 85 & (60 to 90$)$ & 0.372 \\
\hline 6 months & 15 & 89 & (80 to 96$)$ & 23 & 88 & (75 to 95$)$ & 0.598 \\
\hline 12 months & 18 & 85 & (58 to 90$)$ & 21 & 91 & (80 to 95$)$ & 0.122 \\
\hline \multicolumn{8}{|l|}{ E5D } \\
\hline 14 weeks & 13 & 0.73 & (0.6 to 0.8$)$ & 17 & 0.69 & $(0.7$ to 0.7$)$ & 0.450 \\
\hline 6 months & 13 & 0.80 & $(0.7$ to 1.0$)$ & 23 & 0.80 & $(0.7$ to 0.8$)$ & 0.810 \\
\hline 12 months & 19 & 0.85 & $(0.7$ to 1.0$)$ & 21 & 0.85 & $(0.8$ to 1.0$)$ & 0.888 \\
\hline
\end{tabular}

* IQR, interquartile range

$\dagger$ log-rank test

Table VIII. Clinical and dynamometry assessments for the patients who were treated non-operatively six months after their rupture of the tendo Achillis. The missing data sets refer to patients who did not attend for their clinical review

\begin{tabular}{|c|c|c|c|c|}
\hline Variable & Treatment group $(n=15)$ & Control group ( $n=22$ ) & p value ${ }^{*}$ & $95 \% \mathrm{Cl}^{\dagger}$ \\
\hline \multicolumn{5}{|l|}{ Clinical data } \\
\hline Deficit in calf diameter in mm (SD) & $1.37(1.85)$ & $1.11(1.36)$ & 0.634 & -0.82 to 1.32 \\
\hline Deficit in loss of dorsiflexion in degrees (SD) & $-0.7 \quad(7.92)$ & $0.27(5.57)$ & 0.879 & -4.83 to 4.15 \\
\hline Deficit in loss of plantar flexion in degrees (SD) & $4.13 \quad(7.95)$ & $7.27(8.01)$ & 0.248 & -8.57 to 2.29 \\
\hline \multicolumn{5}{|l|}{ Dynamometry data } \\
\hline Deficit in peak concentric torque $(\%)$ & $44(41)$ & 30 (33) & 0.264 & -11 to 39 \\
\hline Deficit in peak eccentric torque (\%) & $45(32)$ & $28(31)$ & 0.110 & -4 to 38 \\
\hline Deficit in mean concentric torque $(\%)$ & $49(36)$ & $35(37)$ & 0.266 & -11 to 39 \\
\hline Deficit in mean eccentric torque (\%) & $50(29)$ & $30(31)$ & 0.067 & -1 to 40 \\
\hline Deficit in total concentric work (\%) & $49(36)$ & $37(36)$ & 0.313 & -12 to 37 \\
\hline Deficit in total eccentric work (\%) & $50(29)$ & $32(29)$ & 0.075 & -2 to 38 \\
\hline
\end{tabular}

$+\mathrm{Cl}$, confidence interval; rank test

work $(p=0.370)$. In the treatment group, ten of the 18 patients who had previously participated in sporting activity did not return to their sport. Similarly, only 11 of 21 patients in the control group returned to their previous sporting activity. Where the number of patients did not equal the total in each group, either the data were not recorded for that patient or the activity was not applicable to that individual. For instance, seven of the treatment group and nine of the control group had retired from their regular job, so no return-to-work data were recorded (Table VI).

The EuroQol and health status questionnaire showed that the patients progressively improved during the course of the study. As expected, the maximum functional deficit occurred 14 weeks after the tendon rupture, two weeks after the patient was mobilised free of their orthosis or cast. Most patients in both groups had returned to their preinjury state by six months after the injury and this improve- 
ment was maintained at one year. Again, where the number of patients did not equal the total in each group these data were not recorded (Table VII).

There was no difference between the groups in the clinical variables or dynamometry measurements at six months (Table VIII).

There was one major complication in the treatment group. This patient sustained a re-rupture of the injured tendon five months after the original rupture when dismounting from his bicycle. He elected to have further nonoperative treatment and made an unremarkable recovery. There were three major complications in the control group. The first patient sustained a re-rupture of the tendon while running for a taxi three months after the initial injury. This patient elected to be treated operatively. At the time of surgery, the re-rupture was found to involve an extensive area of the tendon and a flexor hallucis longus transfer was, therefore, performed at the discretion of the operating surgeon. The second patient showed no signs of tendon healing on clinical or ultrasonographic examination despite prolonged immobilisation in a plaster cast. This patient elected to have no further treatment despite having a permanent defect in her tendon. The third patient had a fatal pulmonary embolism secondary to a deep-vein thrombosis in the injured leg after two weeks of plaster-cast immobilisation.

\section{Discussion}

Early mobilisation with delayed loading has been advocated in a small number of clinical studies. ${ }^{10-13}$ However, there are only two randomised trials in the field.

Mortensen, Skov and Jenson ${ }^{14}$ randomised operativelyrepaired patients to either early, unloaded movement or immobilisation in a plaster cast. They found that early movement shortened rehabilitation time but that unloaded exercise did not prevent muscle atrophy. ${ }^{15}$ Meanwhile, Saleh et $\mathrm{al}^{16}$ found that patients who walked in a custommade orthosis after three weeks in a plaster cast, regained mobility quicker than patients treated for eight weeks in a plaster cast. These reports used weight-bearing mobilisation and movement. Despite the deleterious effects of even short periods of immobilisation, ${ }^{17,18}$ there were at least two weeks between the injury and the use of weight-bearing exercise. Our studies are the first full randomised controlled trials comparing traditional serial plaster casting with immediate loading in an off-the-shelf orthosis.

There are considerable practical advantages for patients in immediate weight-bearing mobilisation. Our studies provide further evidence that accelerated rehabilitation also has functional advantages over traditional plaster-cast immobilisation for patients who have undergone a surgical repair for ruptured tendo Achillis. The patients in the treatment group returned to normal walking and stair climbing much faster than their control group counterparts.

We could not extrapolate this result to a return to sporting activity. In trial 1,16 patients did not to return to their previous sport at all although the majority had good results if assessed by the other outcome measures. However, other factors may have influenced the patients' decision, in particular, the risk of re-rupture. There was also no difference between the groups in terms of return to work. Factors other than the tendo Achillis injury also influenced this variable. Patients in sedentary jobs could usually return to work within a week even in a plaster cast. By chance, there were more manual workers in the treatment than the control group. We advised all patients, in both groups, that they should not drive in either a plaster cast or an orthosis. Consequently, several patients were forced to spend more time away from their jobs than they may have liked, simply because of transport difficulties, although several patients did cycle to work wearing their orthosis. Despite these drawbacks, the return to normal activities, as viewed by the patient, is generally the most important outcome measure. It should, therefore, also be the most important outcome measure for researchers.

Another problem with the trial for surgically-repaired tendon ruptures, was the inclusion of two patients in the treatment group who required augmented tendon repairs. These patients inevitably had considerably slower rehabilitation, which may have adversely affected the group's results. However, the strict intention-to-treat approach adopted by the study removes the risk of bias caused by the exclusion of patients after recruitment.

The EuroQol questionnaire is a generalised health status assessment and would not be expected to show a difference between the groups for an isolated injury to the tendo Achillis. The measurements did show a progressive improvement in both the health status scale (EQol) and the dimensions of health (E5D) score, with the median score for E5D being one or 'normal activity' at one year for both groups.

There are many factors which influence atrophy of the calf muscles after an injury to the tendo Achillis. Calf circumference is an insensitive tool for assessing this and it was, therefore, no surprise to find no difference between the groups in terms of calf muscle circumference six months after injury. The clinical measurements also suggested no difference in range of movement of the ankle. This is reassuring, as it would suggest that no significant tendon lengthening had occurred after immediate weight-bearing mobilisation. Our dynamometry investigations also failed to demonstrate a significant difference between the groups. This outcome measure is attractive as a research tool as it allows quantification of the calf muscle deficit. However, the relationship between controlled dynamometry results and clinical performance has yet to be established.

The complication rate in the treatment group was as important as the primary outcome measure. Both patients who sustained re-ruptures were performing strenuous activities, in breach of the study's protocol. These re-ruptures suggest that patients who are mobilised immediately after a tendo Achillis rupture are more vulnerable to further injury. The ability to bear weight within the orthosis cer- 
tainly encouraged some patients to return to strenuous activity too quickly. The orthosis was chosen for its flexibility. This facilitated a rapid return to a normal gait cycle but, conversely, provided limited support in the event of a fall. Immediate weight-bearing puts greater emphasis on the patient to follow a structured rehabilitation protocol.

In contrast with the trial of immediate weight-bearing in surgically-repaired patients, our study in patients who were treated conservatively provided no evidence of a treatment effect. However, the practical advantages of being able to mobilise fully weight-bearing may be even greater in older patients, who have greater comorbidity.

There was no difference between the non-operatively treated groups in terms of their return to normal activities. More than half of the patients who were involved in sporting activity before their injury decided not to return to their previous sport. This is not surprising, given that many of them had elected to be treated conservatively because they did not plan to continue with impact activities. Also, the data on return to work include only a subsection of the patients as several of them were retired.

Why there should be no difference between the groups in terms of a return to normal walking and stair climbing is less clear, given the significant difference noted in the operatively-repaired group. It may be that the higher number of patients lost to follow-up has created a type 2 error. However, it seems more likely that the treatment effect noted in the patients who underwent operative repair is not present in those managed without surgery.

The theoretical benefits associated with early loading of a healing tendo Achillis are the prevention of detrimental alterations in muscle characteristics and the favourable influence on maturation of collagen fibres within the tendon. ${ }^{4,5}$ If the ends of the tendon are not held in contact by an operative repair, then can the musculotendinous unit really be loaded? The patient may weight-bear through the foot but this load may not be transferred to either the tendon or the gastrocnemius-soleus complex. This may cause the lack of an effect from immediate weight-bearing mobilisation.

Our clinical outcome measures again suggested no treatment effect from the intervention. Reassuringly, there was specifically no difference in the range of dorsiflexion of the ankle between the groups in trial 2 . The range of movement is only a surrogate measurement of tendon lengthening, but the results would indicate that immediate weight-bearing did not produce dramatic stretching within the healing tendon. The dynamometry results also demonstrate no difference between the groups. This may reflect a lack of loading within the gastrocnemius-soleus complex in the patients who were treated non-operatively. However, it may equally reflect the limitations of static dynamometry as a measure of functional performance.

The EQol and E5D measurements showed a progressive improvement in both groups during the study. At one year, the majority of patients had returned to their pre-injury health state. There was no difference between the groups at any stage of the trial.

The complication rate of the patients who were managed non-operatively was also as important as the primary outcome measure. The overall number of complications was low, probably because of the avoidance of surgical risks. There was one re-rupture in each group and a further patient in the control group who did not show any sign of tendon healing in the first place. The risks of re-rupture are known to be higher in patients who are treated non-operatively. ${ }^{18}$

The occurrence of a fatal pulmonary embolism in one of the control group patients should be interpreted with caution. The risks of thromboembolism, and the benefits of early mobilisation in patients with lower-limb trauma have been well documented. However, our study was not designed to address this issue.

It should be stressed that the operative and non-operative arms of this study cannot be compared with each other. They represent two distinct patient populations, with the non-operative group being older. Any comparison would require a further trial.

In summary, our first trial provides further evidence of an improved functional outcome for patients mobilised fully weight-bearing after operative repair of their ruptured tendo Achillis. The two cases of re-rupture in the treatment group suggest that careful patient selection may be required as patients need to follow a structured rehabilitation regimen. The second trial provides no evidence of a functional benefit from immediate weight-bearing mobilisation for conservatively-managed ruptures of the tendo Achillis. However, the practical advantages of immediate weightbearing did not predispose these patients to a higher complication rate. In particular, there was no evidence of tendon lengthening or a higher re-rupture rate. We would, therefore, advocate the use of immediate weight-bearing mobilisation for the rehabilitation of all patients with acute ruptures of the tendo Achillis.

No benefits in any form have been received or will be received from a commercial party related directly or indirectly to the subject of this article.

\section{References}

1. Leppilahti J, Orava S. Total achilles tendon rupture: a review. Sports Med 1998;25: 79-100.

2. Mafulli M. Rupture of the achilles tendon. J Bone Joint Surg [Am]1999;81-A:1019-36.

3. Cetti R, Christensen S, Ejsted R, Jensen NM, Jorgensen U. Operative versus non-operative treatment of achilles tendon rupture. Am J Sports Med 1993;21:791-9.

4. Rantanen J, Hurme T, Kalimo H. Calf muscle atrophy and achilles tendon healing following experimental tendon division and surgery in rats: comparison of postoperative immobilization of the muscle-tendon complex in relaxed and tensioned positions. Scand J Med Sci Sports 1999:9:57-61.

5. Oin L, Appell HJ, Chan KM, Maffulli N. Electrical stimulation prevents immobilisation atrophy in skeletal muscle in rabbits. Arch Phys Med Rehabil 1997;78:512-17.

6. Costa M, Shepstone L, Darrah C, Marshall T, Donell ST. Immediate full-weightbearing mobilisation for repaired achilles tendon ruptures: a pilot study. Injury 2003; 34:874-6.

7. Brooks R. EuroQol: the current state of play. Health Policy 1996;37:53-72.

8. Chester R, Costa M, Shepstone L, Donell ST. The reliability of computer dynamometry as an assessment of recovery following achilles tendon rupture. Foot Ankle Int 2003;24:909-15 
9. Daniel DM, Stone ML, Dobson BE, et al. Fate of the ACL injured patient: a prospective outcome study. Am J Sports Med 1994;22:632-44.

10. Meyer T, Meyer J, Scola E. Achilles tendon rupture: operative tendon suture and semi-functional aftercare with vacuum brace system (Vacoped). Chir Praxis 1998;54:101-8.

11. Saw Y, Baltzopoulos V, Lim A, et al. Early mobilisation after operative repair of ruptured achilles tendon. Injury 1993;24:479-84.

12. Speck M, Klaue K. Early full weightbearing and functional treatment afte surgical repair of acute achilles tendon rupture. Am J Sports Med 1998;26 789-93.

13. Rantanen J, Hurme T, Paananen $\mathbf{M}$. Immobilisation in neutral versus equinus position after achilles tendon repair. Acta Orthop Scand 1993;64:333-5.
14. Mortensen NHM, Skov 0, Jenson PE. Early motion of the ankle after operative treatment of a rupture of the achilles tendon: a prospective, randomized clinical and radiographic study. J Bone Joint Surg [Am] 1999;81-A:983-90.

15. Myerson M. Achillies tendon ruptures. Instr Course Lect 1999;48:219-30.

16. Saleh M, Marshall PD, Senior R, MacFarlane A. The Sheffield split for controlled early mobilisation after rupture of the calcaneal tendon: a prospective, randomised comparison with plaster treatment. J Bone Joint Surg [Br] 1992;74-B:206-9.

17. Haggmark T, Liedberg H, Eriksson E, Wredmark T. Calf muscle atrophy and muscle function after non-operative vs operative treatment of achilles tendon ruptures. Orthopaedics 1986;9:160-4.

18. Lo IKY, Kirkley A, Nonweiler B, Kumbhare DA. Operative versus nonoperative treatment of acute achilles tendon ruptures: a quantitative review. Clin J Sport Med 1997;7:207-11. 